\title{
OR6-5
}

Oral session

\section{Amelioration of antidepressant behavior by Granulocyte colony stimulating factor (GCSF) in depressed rats: Exploring the preclinical role of brain derived neurotrophic factor (BDNF) and neurotransmitters}

\author{
Ajay Prakash ${ }^{1}$, Kanwaljeet Chopra ${ }^{2}$, Bikash Medhi $^{1}$ \\ ${ }^{1}$ Pharmacology, Post Graduate Institute of Medical Education and Research, India, ${ }^{2}$ Neuropharmacology division, \\ University Institute of Pharmaceutical Sciences, Panjab University, Chandigarh, India
}

Objective: This is the first study which explored the role of GCSF in ameliorating the depression like behavior in rat animal model.

Methods: Male adult Wistar rats weighing 200-250 gm were used in the study and were divided into 6 experimental groups, Control group, depression group, GCSF (10\&\#181;g/kg, s.c.) group, GCSF (35\&\#181;g/kg, s.c.) group, GCSF (70\&\#181;g/kg, s.c.) group and fluoxetine group. The interventions were administered as per parallel designed method.

Result: The entry of FST rats in open arm were significantly reduced than the na\&\#239; ve control rats $(p<0.001)$ and entry into the closed arm were significantly increases in FST rats compared to the na\&\#239;ve rats. $(\mathrm{p}<0.001)$ Different doses of GCSF had significantly ameliorate the depression like behavior in day 7 and 14 ( $p<0.05)$; significantly reduced the entry of FST rats in closed arm and correspondingly increases in the open arm entry on day 7 and 14 . $(p<0.001)$ The immobility time of FST rats were significantly increases on day1 in all FST rats whereas after the treatment of GCSF in different doses increases the mobility of rats in FST. $(\mathrm{p}<0.05)$ The GCSF $(70 \& \# 181 ; \mathrm{g} / \mathrm{kg}$, s.c.) was found to be highly significant than other GCSF (10 and 35\&\#181;g/kg, s.c.) treatment in reducing the immobility time in FST performance on day 7 and 14. $(\mathrm{p}<0.05)$ Behavior findings were compared with the direct correlation of 5 -HT and BDNF in the hippocampus of rat brain. The level of 5-HT and BDNF were found to reduce on day 1 after FST i.e.in the depressed rats. The GCSF (10, 35 and 70\&\#181;g/kg, s.c.) treatment significantly increases the level of 5-HT and BDNF on day 14. When the overall comparison was made in the different doses of GCSF, the GCSF ( $70 \& \# 181 ; \mathrm{g} / \mathrm{kg}$, s.c.) was found statistically significant compared to GCSF (10 and $35 \& \# 181 ; \mathrm{g} / \mathrm{kg}$, s.c.) in ameliorating the behavior and physiological improvement of BDNF in FST rats.

Conclusion: In present study we concluded that GCSF significantly improve the antidepressant activity in the depressive rats and also have ameliorative role in neurotransmitters associated with the depression. 